\title{
Restraining order for dendritic cells: all quiet on the fetal front
}

\author{
Rana Chakraborty ${ }^{1}$ and Bali Pulendran ${ }^{2}$
}

1Division of Pediatric Infectious Diseases, Emory University School of Medicine, Atlanta, Georgia, USA.

'Emory Vaccine Center at Yerkes National Primate Research Center, Atlanta, Georgia, USA.

\begin{abstract}
The paradoxical ability to launch effective immunity against pathogens while avoiding the horror autotoxicus of autoimmunity is one of the most remarkable features of the mammalian immune system. This assumes a particular evolutionary significance at the maternal/fetal interface, where avoidance of immune reactivity to the fetus is vital to the propagation of the species itself. Several mechanisms of suppressing maternal immunity against the fetus have been described. The article by Collins et al. in this issue of the JCI describes a novel mechanism of avoiding immune surveillance in which the migratory capacity of dendritic cells at the maternal/fetal interface is restrained (see the related article beginning on page 2062).
\end{abstract}

In eutherian mammals, the implantation of an embryo in a mother's uterus creates an incongruous immunological paradox. The hemochorial placenta provides optimal nourishment and protection to the embryo; however, persistent and intimate contact between the uterine lining (decidua) and the semi-allogeneic fetus (sharing some but not all genes of the mother) should evoke significant immunologic responses akin to a mismatched organ transplant. During pregnancy, however, a seemingly harmonious temporary coexistence of two immunologically distinct multicellular organisms occurs so that the fetus remains protected from any maternal immune responses. Understanding this evolutionary adaptation of immune evasion in the placenta will be pivotal to understanding the induction and maintenance of tolerance from conception to senescence and may provide useful insights into the immunologic role of the placenta during prematurity and recurrent abortions, and during acute and chronic maternal viral infections.

\section{Medawar's postulates}

In 1953 Sir Peter Medawar proposed three general mechanisms of immune evasion within the uterus, which when gravid

Conflict of interest: The authors have declared that no conflict of interest exists.

Nonstandard abbreviations used: LC, Langerhans cell; MHCII, MHC class II.

Citation for this article: J. Clin. Invest. 119:1854-1857 (2009). doi:10.1172/JCI39946. constitutes an immune-privileged site (1). First, there could be induction of immunological tolerance of the mother for fetal antigens. Second, an anatomical and physiological barrier between mother and fetus might prevent access of maternal immune cells to fetal antigens, a mechanism similar to that in effect in other immune-privileged sites, including the testes, eyes, and brain. Third, fetal cells might suppress the expression of alloantigens. These postulates remain plausible but do not explain the paradox of how the maternal immune system acquires unresponsiveness to fetal antigens, while maintaining immune reactivity against infections. A corollary to Medawar's postulates has been put forward to explain this paradox: one that involves site-specific suppression of maternal immune responses at the maternal/fetal interface, thus controlling immune reactivity to the fetus without compromising immunity to pathogens elsewhere (2). The mechanisms underlying site-specific immune suppression facilitating fetal engraftment have been the subject of intense research, and several have been proposed. These include: (i) the expression of FasL by the fetus, which induces Fas-mediated deletion of maternal T cells reactive to the fetus; (ii) expression of nonclassical HLA-G major histocompatibility molecules in cytotrophoblast cells at the maternal/fetal interface, where they maintain a tolerogenic status between the mother and fetus; and (iii) the presence of cells expressing immunosuppressive molecules such as indoleamine 2,3-dioxygenase
(IDO), IL-10, TGF- $\beta$, and $\mathrm{PGE}_{2}$ (3-5). In addition, $\mathrm{CD}^{+} \mathrm{Foxp}^{+}$Tregs, which suppress the activation of the immune system, have been documented to increase in number during early human pregnancy (6).

\section{Maternal/fetal détente}

The study by Collins et al., reported in this issue of the JCI, presents new insights into the role of DCs in mediating this sitespecific suppression (7). DCs represent a rare population of APCs that have long been known to play a key role in sensing pathogens as well as initiating and tuning the quality of the subsequent immune response (8). Like lymphocytes, DCs also comprise distinct cell subsets, which are functionally specialized to produce distinct cytokines that differentially regulate the type of immune response induced. Immature DCs are scattered at the portals of pathogen entry, such as at the mucosal surfaces of the intestine, lungs, and reproductive tracts, and are equipped to sense conserved molecular patterns of pathogens through receptors such as TLRs (9, 10). Upon sensing a pathogen, immature DCs rapidly migrate to the draining LNs and stimulate the naive antigen-specific $\mathrm{T}$ cells, thus launching an immune response. Recent work demonstrates that DCs can also suppress the immune response, through the generation of Tregs, and DCs exhibiting this immunesuppressive property have been observed at mucosal sites such as the gut, lung, and decidua $(9,10)$.

The study by Collins et al. (7) examines the effect of decidualization (encasement of the fetus and placenta in a stromal cell-derived structure called the decidua) on decidual DC migration and function in a murine model of pregnancy (Figure 1). The authors first used flow cytometry to characterize populations of cellular subsets expressing MHC class II (MHCII) in the uterine tissues of virgin mice that had been treated with a high-dose regimen of progesterone designed to simu- 


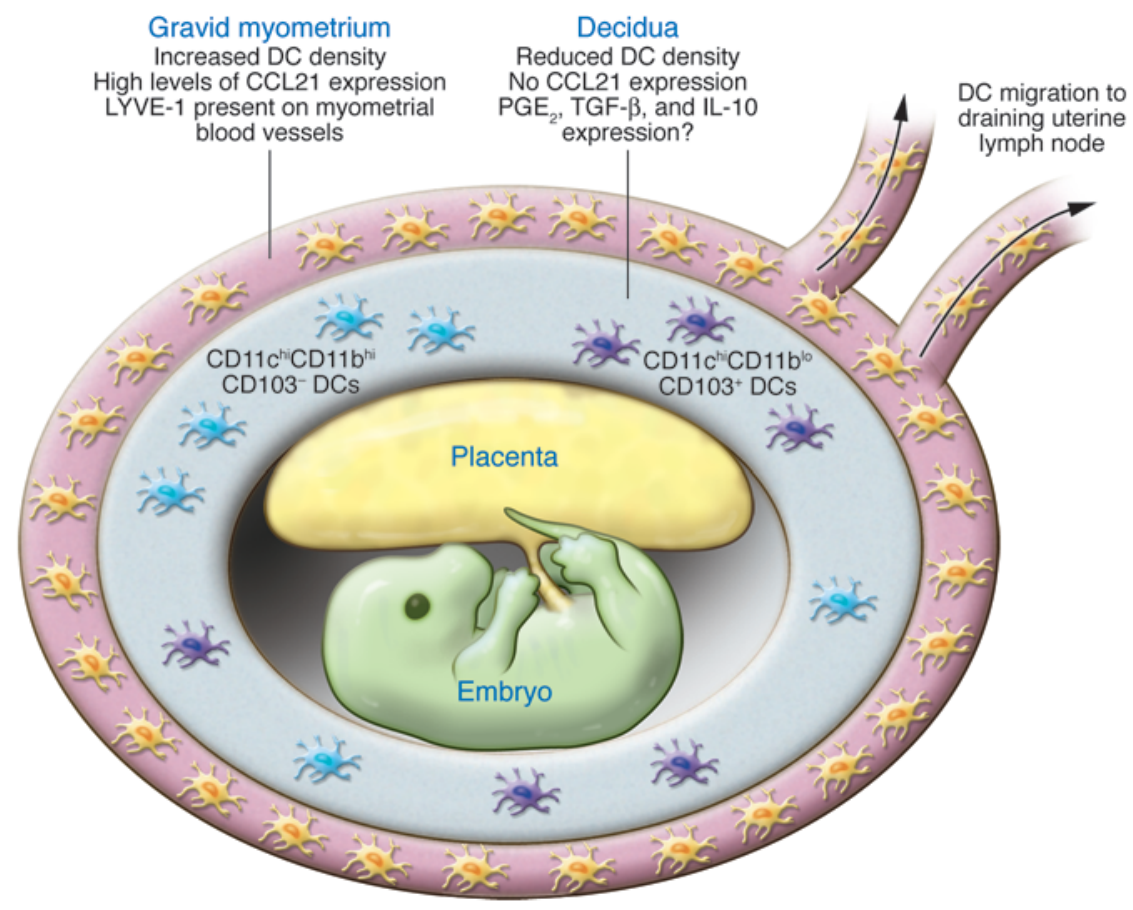

\section{Figure 1}

Hypothetical model of how non-migration of decidual DCs may regulate immune responses directed against the fetus during normal pregnancy. Previous work has shown that lymphatic vessel endothelial receptor-1-positive (LYVE-1+) lymphatic vessels are localized in the myometrium, far away from the maternal/fetal interface upon embryo implantation (12). In their study in this issue of the $\mathrm{JCl}$, Collins et al. (7) demonstrate that the myometrial lymphatic structures were the only structures to express the chemokine CCL21, which is a ligand for CCR7 and drives entry of myometrial DCs into the lymphatic vessels. Furthermore, with uterine growth, DCs in the myometrium increased in tissue density. In contrast, in the decidua there was a reduction in the density of DCs and little or no expression of CCL21. Importantly, the two major DC subsets present in the decidua $\left(C D 11 c^{\text {hi }} C D 11 b^{\text {hi }} C D 103^{-}\right.$and $\left.C D 11 c^{\text {hi }} C D 11 b^{\text {lo }} C D 103^{+}\right)$were unable to migrate from the decidua, even after receiving a potent microbial stimulus such as LPS. These experiments provide evidence of decidual DC entrapment and suggest that a structurally intact decidua appeared to impede the chemokine-directed migration of DCs to the lymphatic vessels of the uterus. This DC entrapment could be the method by which an anti-fetal/placental T cell response is prevented, thus fostering immunological acceptance of the fetus by the maternal immune system.

late pregnancy and suppress the variation in leukocyte numbers that occurs during the estrous cycle. Putative populations of DCs were defined as cells expressing high levels of MHCII and CD11c (an integrin molecule commonly found on murine DCs) and not expressing F4/80 (a molecule commonly found on macrophages). These $\mathrm{MHCII}^{+} \mathrm{CD} 11 \mathrm{c}^{\text {hiF}} 4 / 80^{-}$cells could further be subdivided into $\mathrm{CD} 11 \mathrm{~b}^{\mathrm{lo}} \mathrm{CD} 103^{+}$and CD11bhi CD103- cells (Figure 1), similar to the subsets that have been reported at other mucosal surfaces (10).

To assess whether such DCs could migrate to the draining uterine LNs in WT mice, the authors labeled such DCs in situ by injecting CFSE into the lumen via a nontraumatic transcervical approach and observed the appearance of an $\mathrm{MHCII}^{+} \mathrm{CD} 11 \mathrm{c}^{\text {hi }} \mathrm{F} 4 / 80^{-} \mathrm{CFSE}^{\text {bright }}$ cell population within uterine LNs 28 hours later (7). This population could also be subdivided into $\mathrm{CD} 11 \mathrm{~b}^{\text {lo }} \mathrm{CD} 103^{+}$

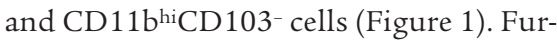

thermore, i.v. injection of a TLR agonist, LPS, a potent stimulus for DC migration and maturation, resulted in enhanced DC migration to the LNs. In contrast to these results in WT mice, DCs in mice lacking the chemokine receptor for CCL21 (CCR7, which is known to guide the migration of DCs from peripheral tissues to the $T$ cell-rich areas of the draining LNs) did not appear to migrate to the uterine LNs, either in the steady state or even in response to LPS.

These observations (7) raised the question of whether such migratory uterine DCs stimulate a $T$ cell response toward the fetus during pregnancy. To address this question, the authors used a model involving transgenic expression of OVA as a surrogate fetal antigen in placental trophoblasts (11). The proliferative response of CFSE-labeled TCR transgenic anti-OVA $\mathrm{T}$ cells adoptively transferred at different stages of gestation demonstrated antifetal/placental $\mathrm{T}$ cell proliferation in the uterine LNs (7). Surprisingly, in CCR7deficient mice, in which DC migration is impaired, the anti-fetal/placental $\mathrm{T}$ cell response was not reduced compared with that in WT mice, suggesting that this response is driven not by DCs emigrating from the maternal/fetal interface, but perhaps by the passive transport of fetal antigen and its uptake by LN-resident DCs. The lack of involvement of DCs in this process contrasts with their established roles as initiators of organ transplant rejection.

\section{DCs on a treadmill}

To understand the apparent lack of involvement of decidual DCs in mediating the anti-fetal/placental $\mathrm{T}$ cell response, the authors evaluated the prevalence and location of these cells in the pregnant uterus (7). With uterine growth, DCs in the myometrium increased in density. However, surprisingly, from E4.5 to E12.5 during pregnancy there was an approximately 
6-fold reduction in the density of DCs in the decidua (Figure 1). Interestingly, WT and $\mathrm{Ccr}^{-1-}$ mice showed similar densities of DCs in both the decidua and myometrium, suggesting that CCR7-dependent cell migration was likely not a key determinant in regulating the densities of DCs at these sites. Previous work has shown that lymphatic vessels positive for lymphatic vessel endothelial receptor-1 (LYVE-1, a type 1 integral membrane glycoprotein expressed on the endothelial cells lining lymphatic vessels) are localized in the myometrium, far away from the maternal/fetal interface, upon embryo implantation (12). In the present study, the authors demonstrate that the myometrial lymphatic structures were the only structures to express the chemokine CCL21 (also known as 6Ckine or SLC), which is a ligand for CCR7 and drives entry into the lymphatic vessels. This raised the possibility that DCs at the maternal/fetal interface would first migrate to the myometrium in order to reach the draining LNs. The authors tested this idea directly by transcervical injection of CFSE as outlined above; however, to ensure that access of CFSE to the decidual tissues was unobstructed by the implanted embryo, the authors used a well-established technique to generate pseudopregnant mice (4) containing artificial decidua. Surprisingly, only a small proportion of DCs in the uterine LNs were CFSE ${ }^{+}$ 28 hours after CFSE injection, and even those few cells were CFSE ${ }^{\mathrm{dim}}$, suggesting that decidual DCs failed to migrate to the LNs. In contrast, myometrial DCs seemed competent to migrate to the draining LNs. Curiously, decidual DCs retained maturation status in situ and migratory capacity toward CCL21 ex vivo when purified cells were subject to Transwell chemotaxis assays, suggesting that there was no intrinsic migratory defect in the decidual DCs. However, these cells remained nonpermissive for cell migration in vivo and in explants even after LPS stimulation and culture in medium containing CCL21. These experiments provide evidence of decidual DC entrapment and also suggest that a structurally intact decidua appeared to impede the chemokine-directed migration of DCs to the lymphatic vessels of the uterus (Figure 1).

\section{Placental particularities}

The findings reported by Collins et al. (7) suggest that this DC entrapment could be a mechanism by which an anti-fetal/ placental $\mathrm{T}$ cell response is prevented and thus fosters immunological acceptance of the fetus by the maternal immune system. These provocative observations raise questions about the mechanisms underlying DC entrapment and their consequences. First, although reduced decidual DC migration is demonstrated, the authors do not address how this could lead to $\mathrm{T}$ cell tolerance (7). Clearly, soluble antigen drains to the LNs via passive antigen transport and can thus be presented to LN-resident DCs. Are these uterine LN DCs tolerogenic? The ability of such LN DCs versus DCs at the maternal/fetal interface to induce $\mathrm{T}$ cell tolerance or Tregs in vitro should be investigated in future studies. Second, by what mechanism does the formation of the decidua restrain DC migration? Is it simply a physical barrier, or is the CCR7dependent chemokine gradient disrupted, perhaps as a result of local production of other regulatory factors within the decidual environment? In this context, the mechanisms controlling the emigration of epidermal Langerhans cells (LCs) after activation include the antiinflammatory cytokines IL-1Ra (13), IL-4 (14), and IL-10 (15), which have been shown to act as negative regulators of migration. Third, are the DCs in the decidua derived from the mother or the fetus? If the latter, are these DCs very immature and therefore functionally incapable of stimulating $\mathrm{T}$ cells, even under strong maturation stimuli? Fourth, if decidual DCs do not migrate to LNs, what is their physiological function? Do they mediate innate responses against infections at the site? In this context, HIV-1, HBV, HCV, herpes, and other viruses have very low rates of transplacental transmission, suggesting that the maternal/fetal interface may nonspecifically restrict viral entry and/or replication (4). Might this be mediated by the innate functions of decidual DCs? Alternatively, by entrapment of decidual DCs, antigen presentation and local viral dissemination may be restricted, which would thereby account for the lack of fetal infection.

These results are reminiscent of an earlier study that showed that the helminth Schistosoma mansoni impairs the migration of LCs from the epidermis to the LNs (16). The inhibitory effect was mediated by parasite-derived $\mathrm{PGD}_{2}$, which specifically impeded the TNF- $\alpha$-triggered migration of LCs through the adenylate cyclase-coupled
$\mathrm{PGD}_{2}$ receptor (DP receptor). This raises the question of whether a similar mechanism involving prostaglandins or other antiinflammatory mediators is responsible for the observed DC entrapment in the decidua. In this context, it is now well appreciated that during normal pregnancy, tolerogenic stimuli from trophoblasts such as progesterone, $\mathrm{PGE}_{2}$, IDO, and vitamin $D$ result in an alternative activation of resident DCs, which results in the establishment of immune tolerance at the maternal/fetal interface (17-19).

In summary, the Collins et al. study (7) highlights an unappreciated mechanism by which the murine decidua inhibits immune surveillance of the maternal/fetal interface. Clinical implications in humans include the potential relevance for immunologic tolerance in mothers with recurrent abortions or premature deliveries. Thus, if the progressive loss of decidual DC tissue density over the first half of postimplantation development and DC entrapment within the decidua represent a normal physiologic state, then migration of human decidual DCs outside the uterus may result in prematurity and recurrent abortions. With further understanding of this process, it may be possible to devise strategies to regulate decidual immunity therapeutically in women with recurrences of these adverse outcomes during pregnancy. Furthermore, such DCs may mediate innate control of viruses such as HIV-1 that have very low rates of transplacental transmission. Future studies examining decidual DCs in humans will surely provide further deep insights into the evolutionary adaptations of the mater$\mathrm{nal} /$ fetal microcosm that are vital to the survival of so many species. As Medawar might have exclaimed, "Fascinating, in every particular!”

\section{Acknowledgments}

The work in the authors' laboratory was supported by funding from the NIH (grants R01 DK057665, R01 AI048638, U19 AI057266, U54 AI057157, N01 AI50019, and N01 AI50025) and from the Bill and Melinda Gates Foundation to B. Pulendran.

Address correspondence to: Bali Pulendran, Emory Vaccine Center at Yerkes National Primate Research Center, 954 Gatewood Road, Atlanta, Georgia 30329, USA. Phone: (404) 727-8945; Fax: (404) 727-8199; E-mail: bpulend@emory.edu. 


\section{commentaries}

1. Medawar, P.B. 1953. Some immunological and endocrinological problems raised by the evolution of viviparity in vertebrates. Symp. Soc. Exp. Biol. 7:320-338.

2. Koch, C.A., and Platt, J.L. 2003. Natural mechanisms for evading graft rejection: the fetus as an allograft. Springer Semin. Immunopathol. 25:95-117.

3. Trowsdale, J., and Betz, A.G. 2006. Mother's little helpers: mechanisms of maternal-fetal tolerance. Nat. Immunol. 3:241-246.

4. Moffett, A., and Loke, C. 2006. Immunology of placentation in eutherian mammals. Nat. Rev. Immunol. 6:584-594.

5. Mellor, A.L., and Munn, D. 2001. Extinguishing maternal immune responses during pregnancy: implications for immunosuppression. Semin. Immunol. 13:213-218.

6. Aluvihare, V.R., Kallikourdis, M., and Betz, A.G. 2004. Regulatory T cells mediate maternal tolerance to the fetus. Nat. Immunol. 5:266-271.

7. Collins, M.K., Tay, C.-S., and Erlebacher, A. 2009.
Dendritic cell entrapment within the pregnant uterus inhibits immune surveillance of the maternal/ fetal interface in mice. J. Clin. Invest. 119:2062-2073.

8. Steinman, R.M., and Banchereau, J. 2007. Taking dendritic cells into medicine. Nature. 449:419-26.

9. Palucka, A.K., Ueno, H., Fay, J.W., and Banchereau, J. 2007. Taming cancer by inducing immunity via dendritic cells. Immunol. Rev. 220:129-150.

10. Pulendran, B., Tang, H., and Denning, T.L. 2008 Division of labor, plasticity and cross-talk between dendritic cell subsets. Curr. Opin. Immunol. 20:61-67.

11. Erlebacher, A., Vencato, D., Price, K.A., Zhang, D., and Glimcher, L.H. 2007. Constraints in antigen presentation severely restrict $\mathrm{T}$ cell recognition of the allogeneic fetus. J. Clin. Invest. 117:1399-1411.

12. Red-Horse, K., et al. 2006. Cytotrophoblast induction of arterial apoptosis and lymphangiogenesis in an in vivo model of human placentation. J. Clin. Invest. 116:2643-2652.

13. Kondo, S., et al. 1995. Interleukin-1 receptor antagonist suppresses contact hypersensitivity. J. Invest.
Dermatol. 105:334-338.

14. Takayama, K., et al. 1999. IL-4 inhibits the migration of human Langerhans cells through the downregulation of TNF receptor II expression. J. Invest. Dermatol. 113:541-546.

15. Wang, B., et al. 1999. Enhanced epidermal Langerhans cell migration in IL-10 knockout mice. J. Immunol 162:277-283.

16. Angeli, V., et al. 2001. Role of the parasite-derived prostaglandin D2 in the inhibition of epidermal Langerhans cell migration during schistosomiasis infection. J. Exp. Med. 193:1135-1147.

17. Munn, D.H., et al. 1998. Prevention of allogeneic fetal rejection by tryptophan catabolism. Science. 281:1191-1193.

18. Hewison M., et al. 2007. Extra-renal 25-hydroxyvitamin D3-1alpha-hydroxylase in human health and disease. J. Steroid Biochem. Mol. Biol. 103:316-321.

19. Saito, S., Tsuda, H., and Michimata, T. 2002. Prostaglandin D2 and reproduction. Am. J. Reprod. Immunol. 47:295-302. 